\title{
Effective transport properties of food products calculated from principles of statistical physics
}

\author{
O. Vitrac $^{\mathrm{a}, *}$ and M. Hayert ${ }^{\mathrm{b}}$ \\ ${ }^{a}$ UMR Fractionnement des Agro-Ressources et Emballage, INRA 614 \\ Moulin de la Housse, 51687 Reims cedex 2, France \\ bUMR Génie des Procédés Environnement et Agro-alimentaire, CNRS 6144 \\ ENITIAA, La Géraudière, 44322 Nantes cedex 3, France
}

\begin{abstract}
This work illustrates how principles of statistical physics including random walks, micro-reversibility and partition functions can be used to derive effective transport properties in complex materials such as food materials. A general formulation based on continuous Langevin Dynamics is proposed to calculate effective diffusivities in emulsions up to the percolation threshold. Interface conditions assuming either reflections or transmissions are used to account for possible partitioning between phases and could be extended to the interactions with macromolecules. The effective properties simulated in 2D emulsions for a wide range of conditions are compared with the analytical core-shell model proposed by Kalnin and Kotomin (1998) for low dense emulsions. The effects were classified in decreasing importance order as follows: ratio of diffusion coefficients, $r_{D}=\left[10^{-2}, 10^{2}\right]$, partition coefficients between the continuous and disperse phases, $K=\left[10^{-2},+\infty[\right.$, globule densities, $d=[0.1,0.4]$, and morphologies. The effects of $r_{D}, K$ and $d$ were nonlinearly coupled in a way that depended on the morphology of the emulsion: heterogeneity in the size of globules, packing of globules, quasi-continuity between globules.
\end{abstract}

Keywords: diffusion coefficient, emulsion, homogenization, statistical physics

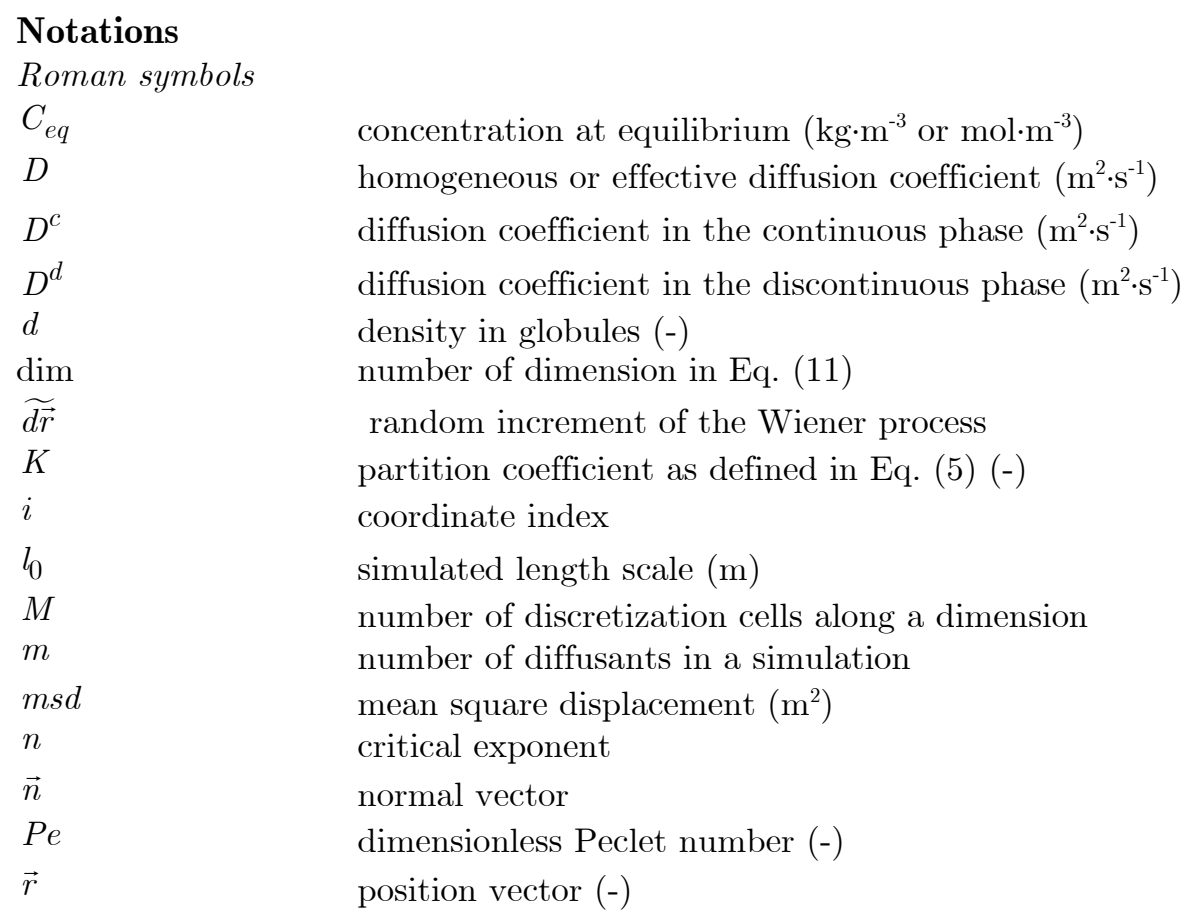

\footnotetext{
Correspondence address: UMR Fractionnement des Agro-Ressources et Emballage, INRA 614, Moulin de la Housse, 51687 Reims cedex 2, France.

Tel +33(0)26918572. Fax : +33(0)26913916. E-mail address: olivier.vitrac@reims.inra.fr
} 


$r_{D}=D^{c} / D^{d}$
$\mathrm{~T}$
$t$
$Z$
$\varnothing$

Greek symbols

$\alpha$

$\delta t$

$\Phi$

$\vec{\xi}$

$\mu_{\varnothing}$

$\sigma \varnothing$

$d \Omega$

Operators and functions

$\begin{array}{ll}\langle\rangle_{x} & \text { average operator over } x \text { values } \\ \delta(x) & \text { delta function of } x \\ \operatorname{pr}(x) & \text { probability of event } x\end{array}$

\section{Introduction}

Foodstuffs consist generally in a mixture of several phases, because their constituents are at different physical states (solid, liquid, gas) or because they have a different chemical affinity (e.g. between lipophilic and hydrophilic constituents). Although Fickian diffusion is strictly limited to ideal mixtures (Nauman and He 2001), it is generally more convenient to represent transport properties even in very complex food by effective diffusion coefficient (Doulia et al. 2000). This approach is physically consistent if both the equivalent transport properties and the equivalent driving force (e.g. concentration gradient) are averaged over a representative volume (Busch and Soukoulis 1995). In practice, this typical volume must be representative of the local composition and detailed microstructure. In particular cases such as in polymer systems or when a specific interaction with a macromolecule is expected, the equivalent description should also take into account small and possibly rare traps, which govern the slowdown of random walks in random environments (Sznitman 2001).

This paper proposes a general methodology based on statistical physics to calculate effective diffusivities of small molecules dispersed at very low concentrations (e.g. additives, active substances, aroma, contaminants...) in simulated or digitized microstructures of foodstuffs. By conception, the formulation accepts i) as many phases as needed and ii) complex thermodynamical descriptions on a wide range of space scales. Since the proposed methodology is a simplified version of Molecular Dynamics, which can be easily updated to multi-rate processes involving several local equilibria of sorption/desorption or association/dissociation, it is thought to be more general than other off-lattice approaches involving lattice gas models (Küntz et al. 2001) or high order spectral approximations (Zhu et al. 2001).

The whole approach is applied to the calculation of effective diffusion coefficients in bi-dimensional emulsions, where the density in globules is up to the percolation threshold and when the ratio between the radius of the smallest and the largest is above $10^{3}$. There is no general model available in the literature to predict effective diffusion coefficients from i) the diffusion coefficients in the continuous and in the discontinuous phase, ii) the partition coefficient between each phase and iii) from the morphology of the emulsion. There is a significant literature on the subject by taking into account only obstacle effects (i.e. impenetrable globules) or by neglecting the possible partition of the diffusing species between both phases (see for example: Riley et al. 1994; Belova and Murch 2003). Earlier results including the Maxwell-Garnett equation are recapitulated in Cranck (1975). The partition effect was first analyzed in details by Ronis (1986). More recently, an algebraic equation, which extends the Maxwell-Garnett equation to cases with partitioning, has been proposed by Kalnin 
and Kotomin (1998) and discussed further in Kalnin et al. (2002). The equation is based on the solution for a core-shell (or coated sphere) model. It takes into account both the effect of partitioning and different transport properties between both phases. In a general case with several globules, the geometric parameter, $\Phi$, in Equation (29) in Kalnin and Kotomin (1998), is however an adjustable parameter. The value of this parameter is expected to vary significantly with the arrangement and morphology of the emulsion. Besides, since the equation is based on a $1 \mathrm{D}$ solution in radial coordinates, some deviations are expected to occur when the connectivity of globules is significant or when globules packing is enough significant to modify the mean free path of particles.

To provide a more general formulation, scaled Langevin dynamics applied to arbitrary microstructures and thermodynamically consistent are developed in the first section. Then the way to determine the effective diffusivities from the mean square distance visited by our "virtual" diffusants is detailed. The following section recapitulates the scaled results obtained for a wide range of conditions including the effect of diffusion coefficients ratio, partition coefficients, emulsion density and emulsion morphology. The reliability of our results is compared to the analytical model of Kalnin and Kotomin (1998).

\section{Langevin Dynamics in emulsions}

\subsection{Equations of motions}

As a result of thermal fluctuations, dispersed molecules at very low concentrations are self-moving particles without interacting with themselves. This transport mechanism that aims at homogenizing the density in dispersed molecules in a homogeneous phase is known as tracer diffusion or molecular diffusion. On a lattice, this random motion on mesoscopic length scales is described with prescribed turning angles. A continuous model in space and discontinuous in time is preferred in this study. The energy required for the motion is randomly pumped in or pumped out the system via a stochastic formulation of equations of motions, which assumes the conservation of momentum and of angular momentum. In absence of external force field, the trajectory of molecules is governed by stochastic differential equations obtained by adding a Gaussian white noise to a first order linear system:

$$
\frac{d}{d t}[\vec{r}(t)]=-\alpha \cdot \vec{r}(t)+\vec{\xi}(t)
$$

where $\alpha$ is a reciprocal correlation time and $\vec{\xi}(t)$ is a random vector with orthogonal components $\xi_{x}(t)$ and $\xi_{y}(t)$. Each component is normally distributed with zero mean and their values are uncorrelated for different times $t$ and $t^{\prime}$, so that they autocorrelation functions obey to the Einstein $\mathrm{s}$ relationship:

$\left\langle\xi_{i}(t) \cdot \xi_{i}\left(t^{\prime}\right)\right\rangle=2 \cdot D_{i} \cdot \delta\left(t-t^{\prime}\right) \quad$ for $i=x, y$

where $D_{i}$ is the diffusion coefficient in the direction $i$ and $\delta(t)$ is the delta or Dirac function.

For $\alpha>0$, the corresponding trajectory includes some low frequency deterministic behavior with a correlation time $1 / \alpha$. Above this correlation time, the trajectory keeps in memory its previous positions (e.g. due to bulk convection).

In practice, Eq. (1) is integrated with a fixed time step, $\delta t$, according to an explicit Eulerian discretization scheme:

$\vec{r}(t+\delta t)=\vec{r}(t)+\delta t \cdot[-\alpha \cdot \vec{r}(t)+\vec{\xi}(t)]$

If $\delta t \ll 1 / \alpha$ so that the diffusion length during $\delta t$ is higher than the typical distance traveled as a result of bulk inertia, the condition of low Peclet values $(P e \ll 1)$ is verified. It entails:

$\vec{r}(t+\delta t)=\vec{r}(t)+\widetilde{d \vec{r}}$

where $\widetilde{d \vec{r}}=\delta t \cdot \vec{\xi}(t)$ is a random increment of the Wiener process, whose orthogonal components are random numbers sampled from a normal distribution with zero mean and a standard deviation defined by $\sqrt{2 \cdot D_{i} \cdot \delta t}$. As a result, the trajectory is obtained from the cumulation of independent increments or moves along each direction. Since all increments increase as $\delta t^{1 / 2}$, the integration scheme defined by Eq. (4) has a global order of 0.5. It is emphasized that this algorithm with low integration order requires long-term iterations to sample the trajectory in space and in frequency. Since only the second moments of the stochastic trajectory is desired, the result is not modified by the initial starting 
positions. A parallel implementation is therefore encouraged by integrating $m$ independent trajectories including each $n$ steps.

Eq. (4) was applied to both the continuous phase, noted "c", and the dispersed phase, noted "d", with a different diffusion tensor (with principal components $D_{x}$ and $D_{y}$ ). In this work, the diffusions coefficients were assumed isotropic so that $D_{x}^{c}=D_{y}^{c}=D^{c}$ and $D_{x}^{d}=D_{y}^{d}=D^{d}$.

\subsection{Interface conditions in biphasic systems}

In a biphasic medium, additional assumptions are required to account for possible different chemical affinities of the diffusant for phases $\mathrm{c}$ and $\mathrm{d}$. If sorption and desorption processes are reversible and conveniently approximated by a Henry like relationship in each phase, the macroscopic equilibrium between c and $\mathrm{d}$ is completely determined by a partition coefficient, $K$, defined by:

$$
K=\frac{C_{e q}^{c}}{C_{e q}^{d}}
$$

where $\left\{C_{e q}^{k}\right\}_{k=c, d}$ is the concentration of diffusants in the phase $k$ at equilibrium. Concentration are expressed in amount (mass or number of molecules) per volume unit so that there are related to the density in molecules in each phase.

If the trajectories are calculated at equilibrium, they shall verify the condition of local thermodynamical equilibrium (LTE) at the c-d interface, noted $d \Omega$. At each simulated time, LTE assumes that particles are exchanged across the interface so that the ratio of occupancy probabilities on both sides of the interface is also controlled by the value of the partition coefficient, which may be measured macroscopically. Formally, the macroscopic partition coefficient is connected to the partition functions in each phase, noted $\left\{\left.Z^{k}\right|_{d \Omega}\right\}_{k=c, d}$, via:

$K=\frac{\left.Z^{c}\right|_{d \Omega}}{\left.Z^{d}\right|_{d \Omega}}$

It is worth to notice that both partition functions in eq. (6) are assumed to be renormalized in such a way that they are intensive quantities, which are proportional to the occupancy probabilities on both sides of the interface. The origin of the partitioning is an energy barrier (transition state), which must be overcame to hop from one medium to another. In our formulation, the partition function at the saddle point (transition state) remains unknown and only the equilibrium probabilities on both side the interface are consistently described. As a result, the frequencies of crossing in the directions $c \rightarrow d$ and $d \rightarrow c$ are arbitrary and controlled by our time discretization scheme. For a particle close to the interface, the probability of crossing the interface in the direction $c \rightarrow d$ and in the direction $d \rightarrow c$, noted $\operatorname{pr}(c \rightarrow d \mid d \Omega)$ and $\operatorname{pr}(d \rightarrow c \mid d \Omega)$, are, at any time, given by

$$
\left\{\begin{array}{l}
\operatorname{pr}(c \rightarrow d \mid d \Omega)=\frac{\left.Z^{d}\right|_{d \Omega}}{\left.Z^{c}\right|_{d \Omega}+\left.Z^{d}\right|_{d \Omega}}=\frac{1}{1+K^{-1}} \\
\operatorname{pr}(d \rightarrow c \mid d \Omega)=\frac{\left.Z^{c}\right|_{d \Omega}}{\left.Z^{c}\right|_{d \Omega}+\left.Z^{d}\right|_{d \Omega}}=\frac{1}{1+K}
\end{array}\right.
$$

Since $\operatorname{pr}(c \rightarrow d \mid d \Omega)+\operatorname{pr}(d \rightarrow c \mid d \Omega)=1$, Eq. (7) verifies the principle of mass conservation on both sides of the interface. For consistency, when the particle does not cross the interface, it is assumed to stay in the initial phase, so that:

$$
\left\{\begin{array}{l}
\operatorname{pr}(c \rightarrow c \mid d \Omega)+\operatorname{pr}(c \rightarrow d \mid d \Omega)=1 \\
\operatorname{pr}(d \rightarrow d \mid d \Omega)+\operatorname{pr}(d \rightarrow c \mid d \Omega)=1
\end{array}\right.
$$


When they are applied with a constant time step of integration, it is straightforward to demonstrate that Eq. (7) and (8) comply the principle of detailed balance in the direction normal to the interface, $\vec{n}_{d \Omega}$ :

$$
\underbrace{\frac{1}{\delta t} \cdot[\operatorname{pr}(c \rightarrow c \mid d \Omega)+\operatorname{pr}(d \rightarrow c \mid d \Omega)] \cdot \vec{n}_{d \Omega}}_{\text {normal probability flux towards the c side }}=\underbrace{-\frac{1}{\delta t} \cdot[\operatorname{pr}(d \rightarrow d \mid d \Omega)+\operatorname{pr}(c \rightarrow d \mid d \Omega)] \cdot \vec{n}_{d \Omega}}_{\text {normal probability flux towards the d side }}
$$

The detailed balance condition, also known as microreversibility, is a sufficient, but not necessary, condition to ensure a Gibbsian equilibrium state (Marsh and Coveney, 1998). For stability and convergence purposes in the integration scheme, stronger constraints are applied at the interface by forcing the time-reversibility of the trajectory for the four possible transitions at the c-d interface: $c \rightarrow c|d \Omega, \quad d \rightarrow d| d \Omega, \quad c \rightarrow d \mid d \Omega$ and $d \rightarrow c \mid d \Omega$. Since the current transport equations do not conserve the energy, the proposed interface conditions allow also to dissipate energy. The applied boundary conditions are illustrated on practical cases in Fig. 1 and detailed as follow.

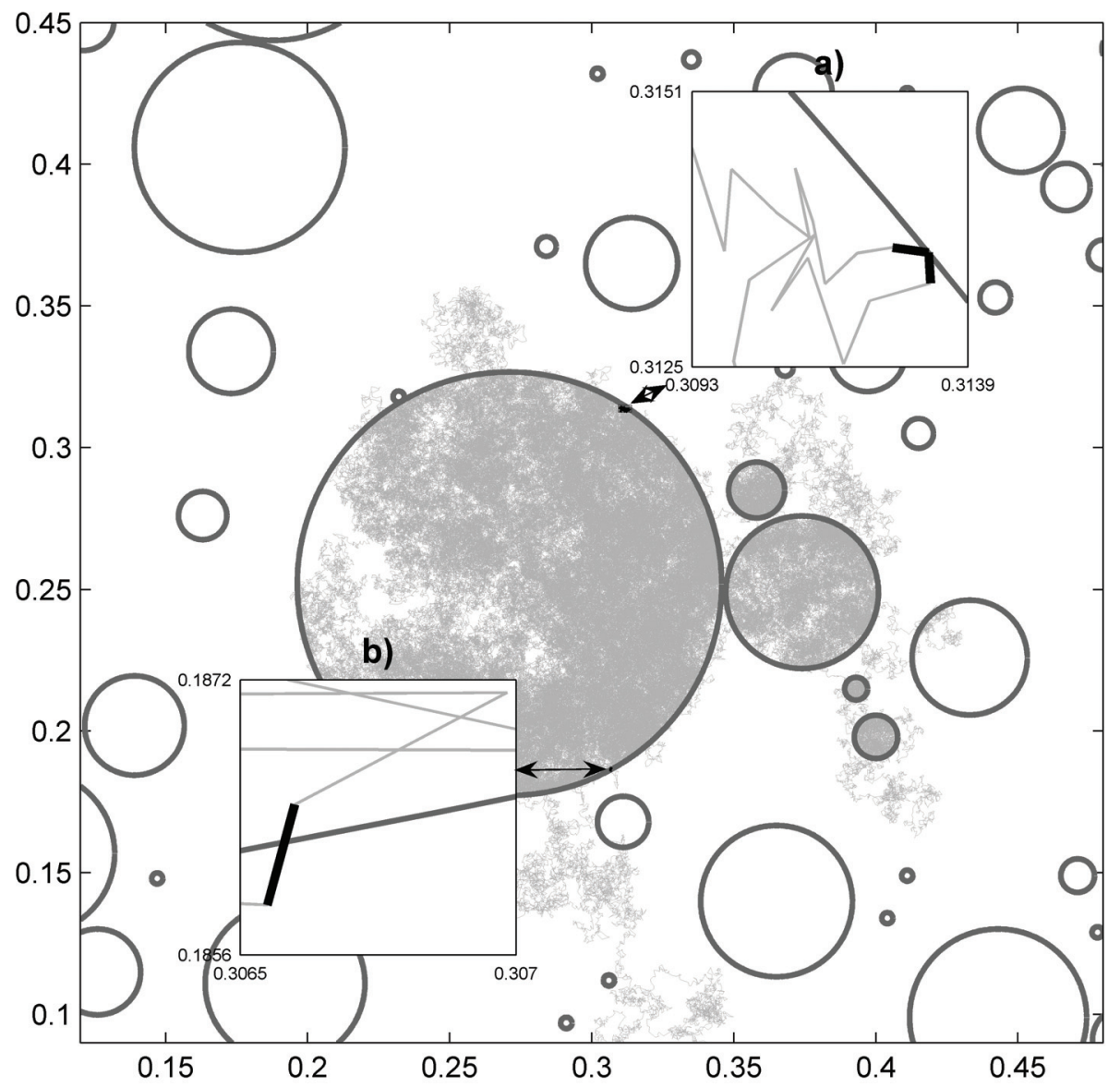

Fig. 1. Illustration of interface conditions: a) reflection $\left(\left.d \rightarrow d\right|_{\partial \Omega}\right)$, b) transmission $\left(\left.d \rightarrow c\right|_{\partial \Omega}\right)$. The depicted condition correspond to $K=10, r_{D}=2, d=0.3$. The positions of both inserts are identified by double arrows.

\subsubsection{Reflective boundary conditions for transitions $c \rightarrow c \mid d \Omega$ and $d \rightarrow d \mid d \Omega$}

A specular reflective boundary condition was applied to the "internal" transitions $c \rightarrow c \mid d \Omega$ and $d \rightarrow d \mid d \Omega$. During $\delta t$, the local trajectory before and after the collision with the interface at position I was therefore microreversible and symmetric to $\vec{n}_{d \Omega}$.

2.2.2 Transmitting boundary conditions for transitions $c \rightarrow d \mid d \Omega$ and $d \rightarrow c \mid d \Omega$

When the trajectory crosses the interface in straight line at a point I from a point A to a point B, the time-reversibility principle implies that the magnitude of the normal flux at I, which is generated by 
particles emitted by A and reaching B, is the same as the one generated by particles emitted by B and reaching A. Since the random increments, $\widetilde{d \vec{r}}$, are not equivalently distributed between each phases, the distance IB after the interface (for $\|\widetilde{d \vec{r}}\|>A I$ ) must be rescaled to verify similar probabilities for the transitions $\mathrm{A} \rightarrow \mathrm{B}$ and $\mathrm{B} \square \mathrm{A}$ during $\delta t$. Since $\widetilde{d \vec{r}}$ increases as the square root of the diffusion coefficient, IB is obtained by:

$I B=\sqrt{\frac{D^{a}}{D^{b}}} \cdot A I$

where $D^{a}$ and $D^{b}$ are the diffusion coefficients in the positions A and B respectively.

It follows from Eq. (10) that only the direction of $\overrightarrow{\mathrm{A} B}$ and the position of the interface crossing are chosen randomly at time $t+\delta t$. The distances IA and IB depend on the previous position at time $t$ and on the local environment (closeness of interfaces).

When IB is higher than the distance between objects or higher than the distance between the interfaces within a same object, the ballistic trajectory is recursively segmented into subsequent reflections and transmissions which follow the rules detailed in 2.2.1 and 2.2.2. In the current formulation, a maximum of reflections was set to 10. The simulation was scaled (see 3.2) so that more than $95 \%$ of mean free paths (ballistic lengths visited during $\delta t$ ) were at least 10 folds smaller than the typical distance between objects and than the characteristic length of the smallest objects. This condition enforced that the sizes of considered mesoscopic objects (globules in this work) were much larger than the size of solvent molecules and which were responsible for the random trajectories of particles at molecular level.

\section{Simulated conditions}

\subsection{Scaled 2D emulsions}

$2 \mathrm{D}$ emulsions were represented as randomly dispersed globules, possibly in contact, on a scaled periodic cell on $\left[0,1\left[\times\left[0,1\left[\right.\right.\right.\right.$, corresponding to an infinite medium of periodicity $l_{0}$. The size of each globule was sampled from a log-normal distribution with arithmetic average, $\mu_{\varnothing}$, and standard deviation, $\sigma_{\varnothing}$. The positions of each globule were fixed during dynamics. The effect of globule dynamics is discarded since its time constant is expected to be much higher than the dynamics of diffusants.

The tested conditions are summarized in Table 1. Two extreme morphologies were studied: a monodisperse emulsion, denoted $\mathrm{E}_{0}$, and a very heterogeneous emulsion, denoted $\mathrm{E}_{1}$. The effect of tortuosity was tested by varying the density, $d$, between 0.1 and 0.4 . To reduce possible bias due to the construction of emulsions, all simulations were performed on a small set including between 4 and 7 sampled structures. According to the set density, each morphology included between 70 and 800 globules.

Table 1. Tested morphologies of emulsions.

Mean and standard deviations are based on sampled values.

\begin{tabular}{c|cccccc}
\hline \multirow{2}{*}{ morphology } & \multicolumn{2}{|c}{ droplet diameter $\left(\times l_{0}\right)$} & \multicolumn{3}{c}{ number of repetitions } \\
\cline { 2 - 6 } & $\mu_{\varnothing}$ & $\sigma_{\varnothing}$ & $\mathrm{d}=0.1$ & $\mathrm{~d}=0.2$ & $\mathrm{~d}=0.3$ & $\mathrm{~d}=0.4$ \\
\hline $\mathrm{E}_{0}$ & $4 \cdot 10^{-2}$ & $3 \cdot 10^{-8}$ & 4 & 4 & 4 & 4 \\
$\mathrm{E}_{1}$ & $2 \cdot 10^{-2} \pm 4 \cdot 10^{-3}$ & $2.8 \cdot 10^{-2}$ & $\pm 6 \cdot 10^{-3}$ & 7 & 7 & 7 \\
7
\end{tabular}


Examples of emulsions and their corresponding size distributions are given in Fig. 2.
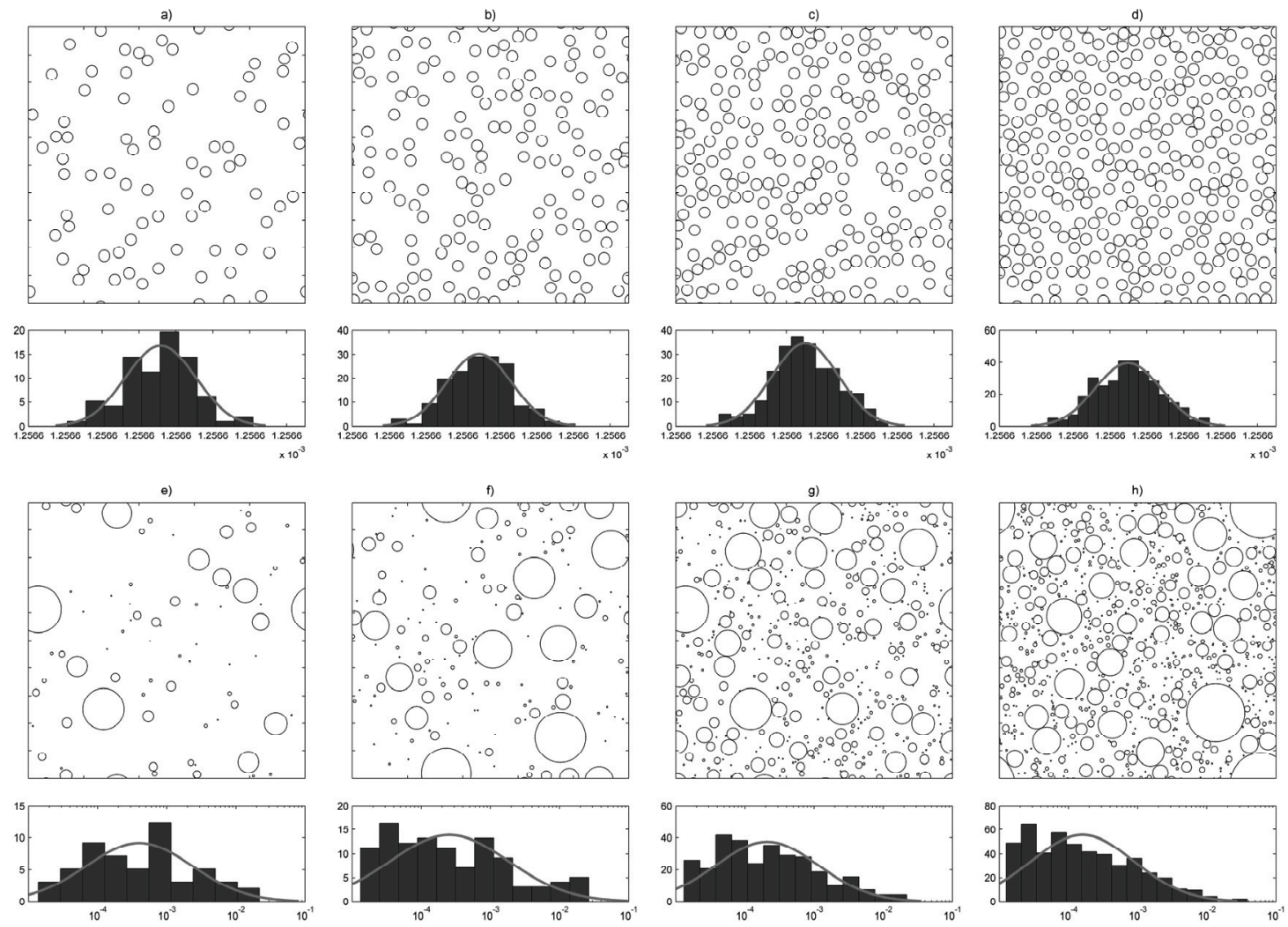

Fig. 2. Typical $2 \mathrm{D}$ emulsions on a $[0,1] \times[0,1]$ domain for a,e) $d=0.1, \mathrm{~b}, \mathrm{f}) d=0.2, \mathrm{c}, \mathrm{g}) d=0.3$

and $\mathrm{d}, \mathrm{h}) d=0.3$; a-d) homogeneous emulsions $\left(\mathrm{E}_{0}\right)$, heterogeneous emulsions $\left(\mathrm{E}_{1}\right)$. Histograms plot the distributions of surface area of droplets fitted with a continuous normal and a log-normal distribution for $\mathrm{E}_{0}$ and $\mathrm{E}_{1}$ respectively.

\subsection{Tested range of physico-chemical properties}

The diffusion coefficient in the dispersed phase, $D^{d}$, was arbitrary chosen as reference. Its value was taken lower than $l_{0}^{2} \cdot\left(4 \cdot M^{2} \cdot \delta t\right)^{-1}$ so that more than $95 \%$ of elemental trajectories during $\delta t$ had a length lower than the step of the fine grid. This condition ensured that even small globules were correctly described. The granularity, which was thus obtained, was far beyond possibilities of discretization of low order spectral methods such as finite elements.

The simulations were performed for different ratios $r_{D}=D^{c} / D^{d}$ between $10^{-2}$ and $10^{2}$. This range was assumed to fit with generally needs. By contrast, partition coefficients, $K=0$, were tested on the whole scale from 0 (no affinity for the continuous phase) to $K \rightarrow \infty$ (no affinity for the dispersed phase). A particular care was brought to the developed code to verify the equilibrium properties defined by Eq. (7) even for the most extreme conditions. Reliable results were obtained by describing accurately multiple reflections in dense regions or within small globules. In practice, a search procedure including three levels was used to track interfaces.

\subsection{Identification of diffusion coefficients}


In absence of confinement on long time scales, the diffusion is expected to be linear in the long time limit and large length scales (i.e. larger than the typical length of globules). This limit hydrodynamic regime implies that the mean square displacement is proportional to the diffusion time, $\mathrm{T}$ :

$D=\frac{1}{2 \cdot \operatorname{dim}} \cdot \lim _{\mathrm{T} \rightarrow \infty} \frac{\partial}{\partial \mathrm{T}}\left\langle\left\|\vec{r}_{(t+T)}-\vec{r}_{(t)}\right\|^{2}\right\rangle_{t, i=1 \ldots m}$

Eq. (11), known as Einstein equation, relies on the central limit theorem and assumes that the trajectory observed during the typical time scale $\mathrm{T}$ is centered on average on all possible starting positions $\vec{r}_{(t)}$. dim is the number of dimensions. \langle\rangle$_{t, i=1 \ldots m}$ is the average operator over all possible initial times and diffusing molecules. Since momentum is conserved in Langevin dynamics, the center of mass chosen as reference frame is fixed and no correction due to a possible moving reference frame (e.g. flowing emulsion) is required. It is however assumed that the typical time scale responsible for the modification of the distances between globules is longer than the time scale required to reach the asymptotic regime.

For the particular value $K=0$, a confinement in the dispersed phase occurs over all time scales. The mean square displacement or equivalently the total variance of the position vector converges towards the maximum surface of the dispersed phase $d \cdot l_{0}^{2}$. The equivalent diffusivity is zero since no transport between globules is allowed. In practice, a non-zero diffusion coefficient was achievable even for low $K$ values when a sufficiently large number of $c \rightarrow d \mid d \Omega$ and $d \rightarrow c \mid d \Omega$ transitions were sampled.

\section{Results and discussion}

\subsection{Typical trajectories and mean-square displacements}

\subsubsection{Trajectories}

The effect of $r_{D}$ and $K$ on the trajectory of $m=5$ diffusants is depicted in Fig. 3 for $r_{D}=2$ and $K=\{0.1,1,10\}$ in an heterogeneous emulsion with $d=0.3$. The corresponding cumulated diffusion time was set to $m \cdot t=5 \cdot 10^{6} \cdot \delta t$ to get a partly ergodic system but without reaching the equilibrium. As a result, the distribution of corresponding residence times were not uniform in each phase and the details of the trajectories were discernible. Residence times averaged over all diffusants were calculated on a $50 \times 50$ grid and plotted as filled regions with iso-residence times based on $2 \mathrm{D}$ cubic interpolants.

Raising the affinity for the dispersed phase $(K \rightarrow 0)$ led to a winding of the trajectories (Fig. 3a) inside the globules and increased the residence time in the globules (Fig. $3 \mathrm{~b}$ ). For $K=1$, the trajectories were spread over all the simulated domain without any distinction between both phases (Fig. 3c). In details, the residence times looked higher inside the globules than in the continuous phase (Fig. 3d). This effect is related to a twice lower diffusion coefficient in the disperse phase. It is however observable only in the transient regime when the simulation time is insufficient to sample the longterm trajectory in the continuous phase. Indeed, the number of recirculation is expected at equilibrium to be twice in the continuous phase than in the dispersed phase so that the residence times between both phases are equal at equilibrium. The situation $K=10$ led to a more rapid uniform filling of the continuous space between globules (Fig. 3e) but with a low visiting rate inside globules (Fig. 3f). It is however underlined that the transient trajectories appeared also concentrated between globules, particularly in regions were the density in globules was high or led to tortuous paths.

From previous examples, several phenomena are responsible for a transient confinement of the trajectories either globules or between globules: i) local low diffusion coefficients, ii) effect of obstruction between locally packed globules and iii) high affinity for the dispersed phase. All phenomena may be combined and act together to lower the mean square distance visited by the diffusant and consequently the asymptotic diffusion coefficient. However, although they lead to similar effects on short terms, differences arise from the persistence of the confinement on long time scales. As a result, agonist and antagonist effects on confinement can be expected according to the considered $r_{D}$ and $K$ values, the density and morphology of the emulsion. 

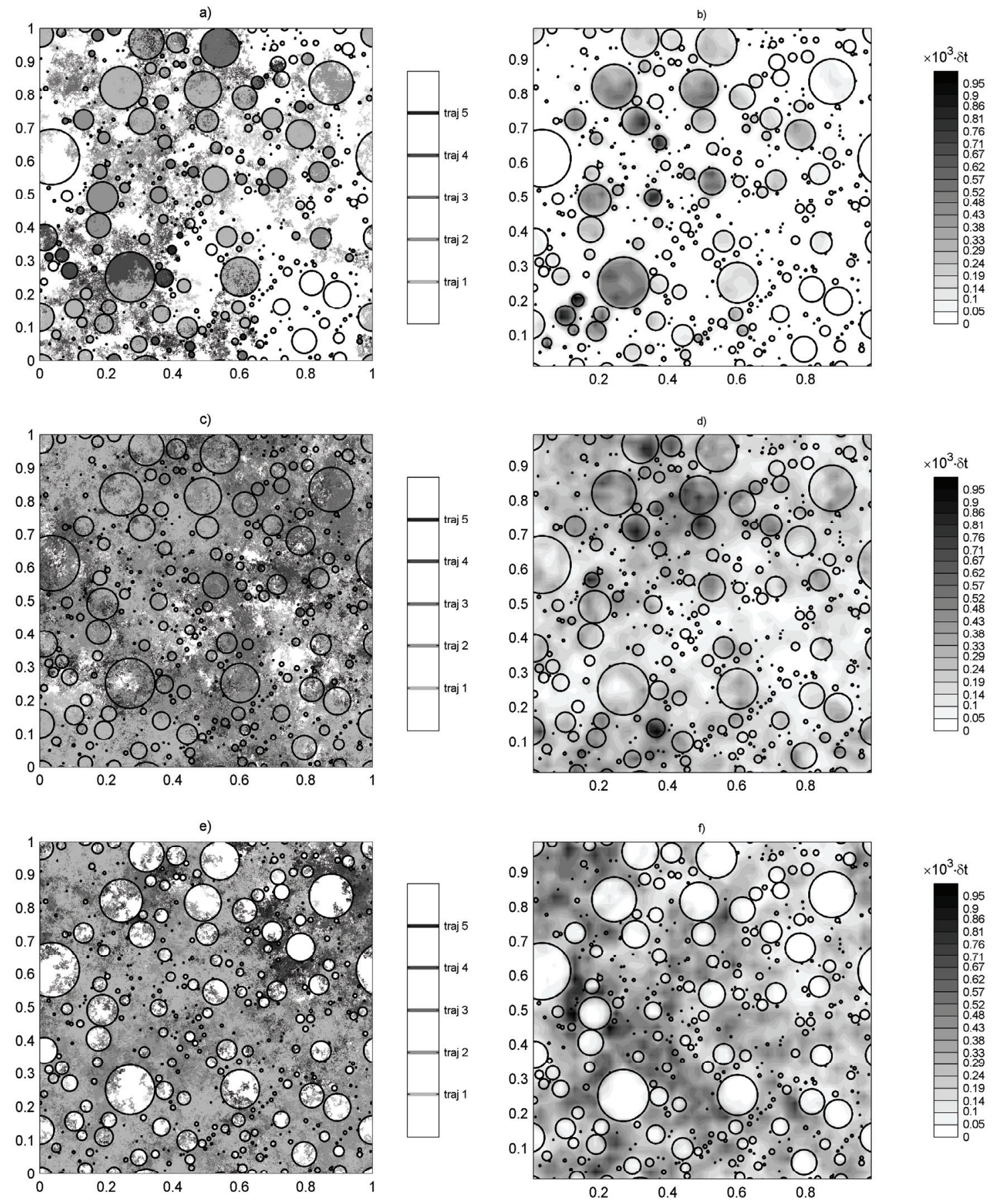

Fig. 3. a,c,e) Typical trajectories during $10^{6} \cdot \delta t$ and b,d,f) corresponding residence times in the emulsion plotted in Fig. $2 \mathrm{~g}(d=0.3)$ for $r_{D}=2$ and a-b) $K=0.1$, c-d) $K=1$, e-f) $K=10.5$ independent trajectories are plotted, residence times are integrated over all 5 trajectories and calculated on a on a $50 \times 50$ grid $($ step size $=0.02)$. 


\subsubsection{Mean-square displacements}

The typical dimensionless mean-square displacements (msd) versus the dimensionless diffusion time $\left(t \cdot D^{d} \cdot l_{0}^{-2}\right)$ are plotted for a counter-intuitive situation: $E_{1}, d=0.3$ and $r_{D}=0.1$ and wide range of $K$ values in Fig. 4. The msd values of each curve were averaged over the trajectories of 100 diffusants. The theoretical curves corresponding to a scaled asymptotic diffusion coefficient, $D / D^{d}$, equals to the scaled diffusion coefficient in the continuous phase, $r_{D}$, are also indicated. On a log-log scale, the asymptotic regime is identified by an unitary slope and the corresponding diffusion coefficient is given by its intercept.

In the depicted situation, the transport in the continuous phase is the limiting factor on short time scales so that msd values are lower when the affinity for the dispersed phase (low $K$ values) is higher. By contrast, on long time scales and for low $K$ values, the trend is reverse and the limiting factor is the confinement in the dispersed phase. The change in behavior is identified by a change in the slope of the scaling relationship of msd with diffusion time. For all morphology, there is always an optimal $K$ value, lower than 1 , which leads to the shortest paths between all possible combinations on short and long time scales. This value is all the lower than $d$ is higher.

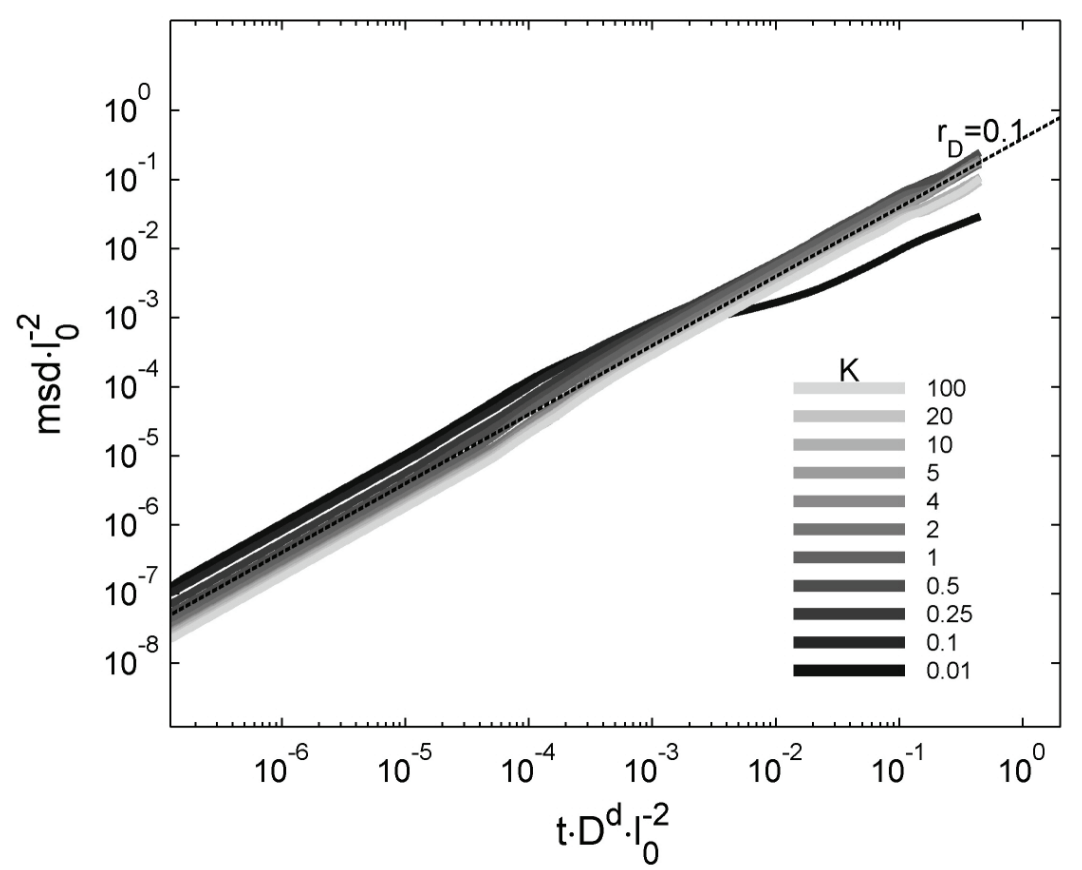

Fig. 4. Scaling relationship of msd with diffusion time for $E_{1}, d=0.3$ and $r_{D}=0.1$.

\subsection{Variation of the asymptotic diffusion coefficient with studied properties (2042 tested conditions)}

The effects of all tested parameters were analyzed as least-square bi-variate cubic spline approximations of $D / D^{d}$ versus $r_{D}$ and $K$ for all tested $d$ values and morphologies. Each model consisted in 4 continuous piecewise approximations along each variable $\left(r_{D}\right.$ and $K$ ). The positions of nodes were optimally calculated to minimize the deviation to calculated results. Boundary conditions were applied to fit asymptotic behavior for $r_{D}=\{0,+\infty\} \times K=\{0,+\infty\}$. The 8 derived abacuses are plotted in Fig. 5 as contour plots for common $D / D^{d}$ values.

The contour plots exhibit a half square shape which confirms the predominance of $r_{D}$ effects for $K$ values higher than 1. For a same $r_{D}$ value, a variation of $K$ between $10^{-2}$ and $+\infty$ generates a variation in of $D / D^{d}$ up to 5 folds. A greater effect is expected for values lower than $10^{-2}$. 
The effect of $K$ is significant mainly for values lower than 1 . When the diffusivity is higher in the discontinuous phase, the asymptotic diffusion coefficient does not vary monotonously with $K$ (Fig. $5 \mathrm{~d}$ ) and reaches a maximum close to $K=1$. The maximum is only located at $K=1$ only for $r_{D}=1$ (Fig. 5e). It is obtained for $K$ values slightly lower than 1 for $r_{D}<1$ and above 1 for $r_{D}>1$ (Fig. $5 \mathrm{f}$ j). $K \rightarrow \infty$ yields never a maximum.

Oscillations observed for certain contours are not an artifact and are related to non-linear combined effects between $r_{D}$ and $K$. They are connected to non monotonous variations of $D / D^{d}$ with $K$ for $r_{D}$ values around 1 . The effects of $d$ and of the morphology consist mainly in modifying the location of this maximum on the $K$ scale and consequently the amplitude of the oscillations.
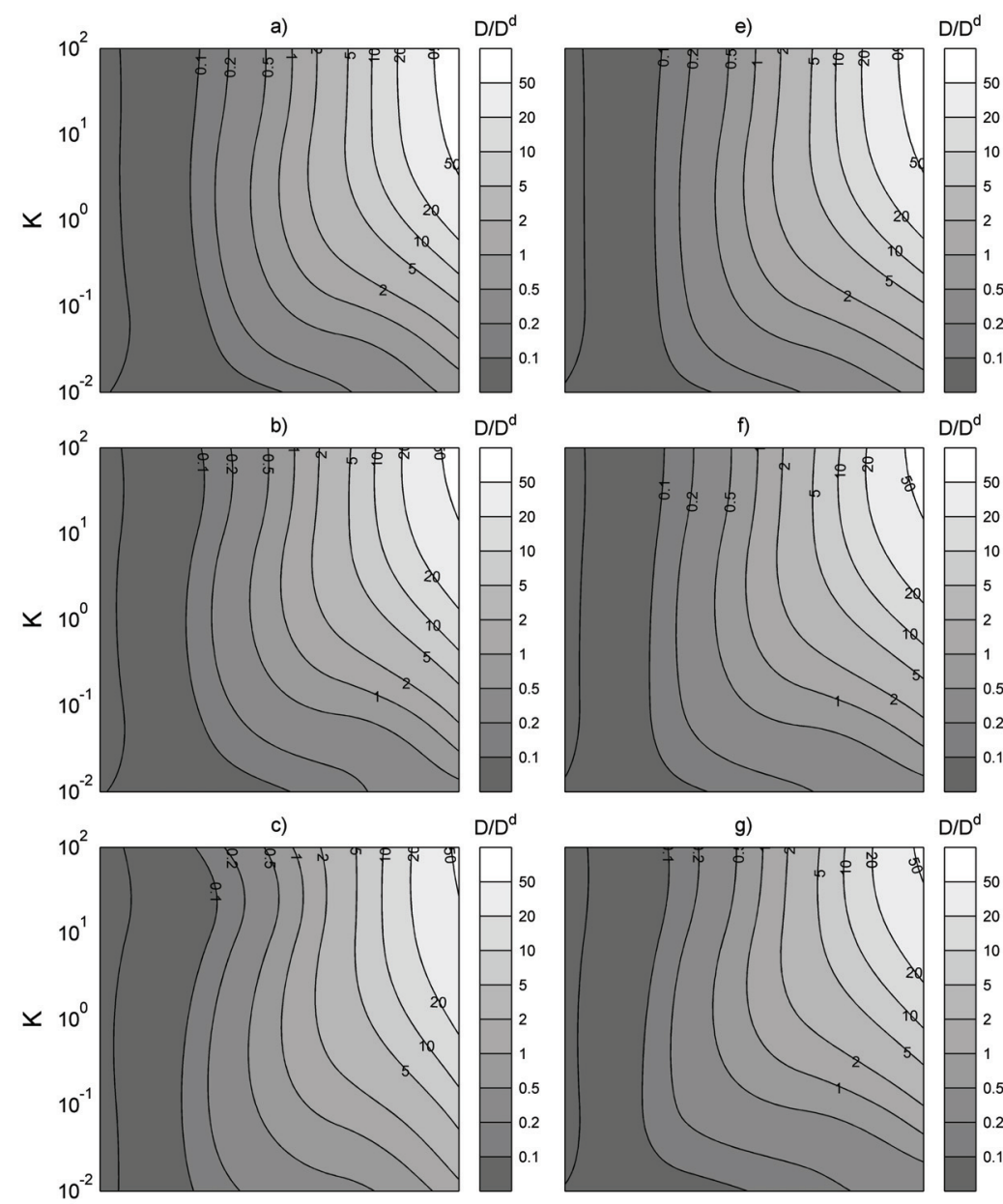

$\mathrm{D} / \mathrm{D}^{\mathrm{d}}$
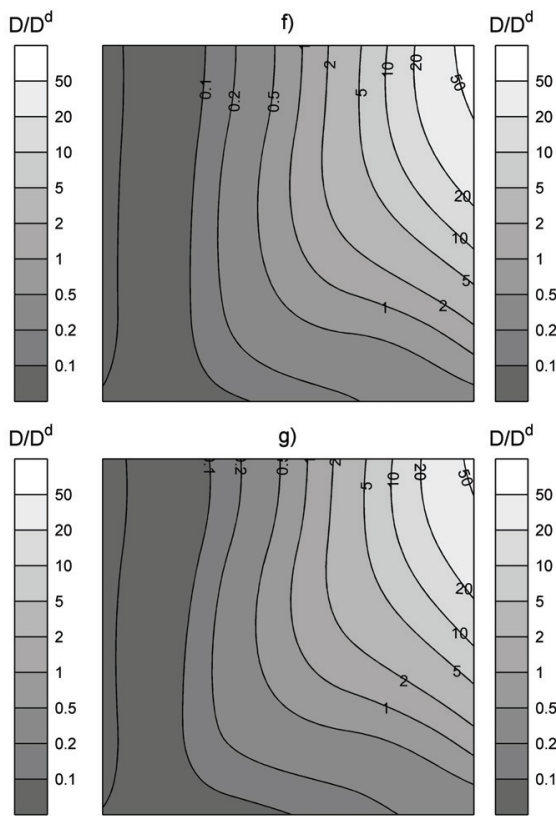

$\mathrm{D} / \mathrm{D}^{\mathrm{d}}$
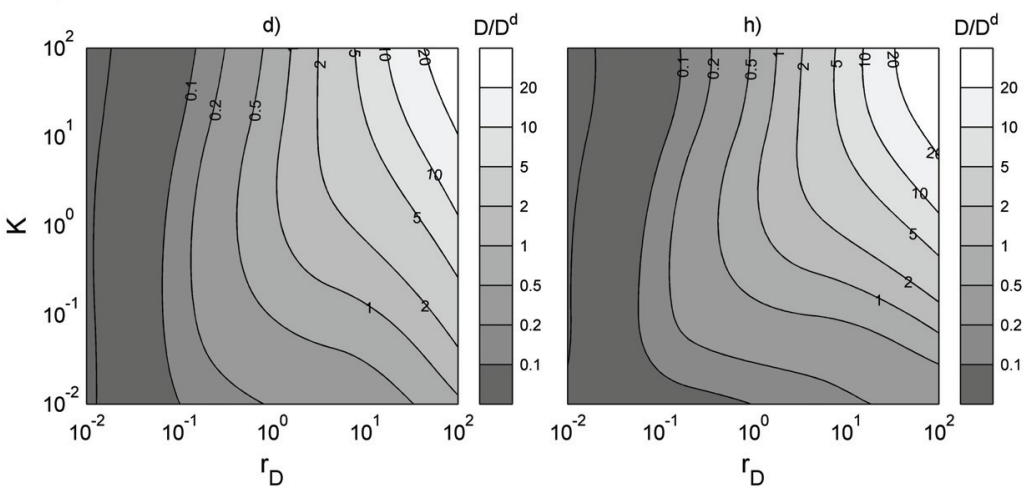

Fig. 5. Bi-variate cubic spline approximations of the asymptotic diffusion coefficient with $K$ and $r_{D}$ for a,e) $d=0.1, \mathrm{~b}, \mathrm{f}) d=0.2, \mathrm{c}, \mathrm{g}) d=0.3, \mathrm{~d}, \mathrm{~h}) d=0.4$, a-d) morphology $E_{0}$, e-h) morphology $E_{1}$. 
In our notations, Eq. (29) in Kalnin and Kotomin (1998) is written in 2D:

$$
\frac{D}{D^{d}}=\frac{r_{D}}{1-\Phi-\frac{\Phi}{K}} \cdot\left[1+2 \cdot\left(\frac{1}{K}-r_{D}\right) \cdot \frac{\Phi}{r_{D}+\frac{1}{K}-\left(\frac{1}{K}-r_{D}\right) \cdot \Phi}\right] \text { with } 0 \leq \Phi \leq 1
$$

Eq. (13) admits a free parameter, $\Phi$, which must be fitted to either experimental or simulated results to predict the effective diffusion coefficient in emulsions. Since, it is originally related to the volume ratio of concentric regions, $\Phi$ was first fitted to results obtained for a same $d$ value and morphology.

The results are depicted in Fig. 6 for all conditions reliably tested in this study.

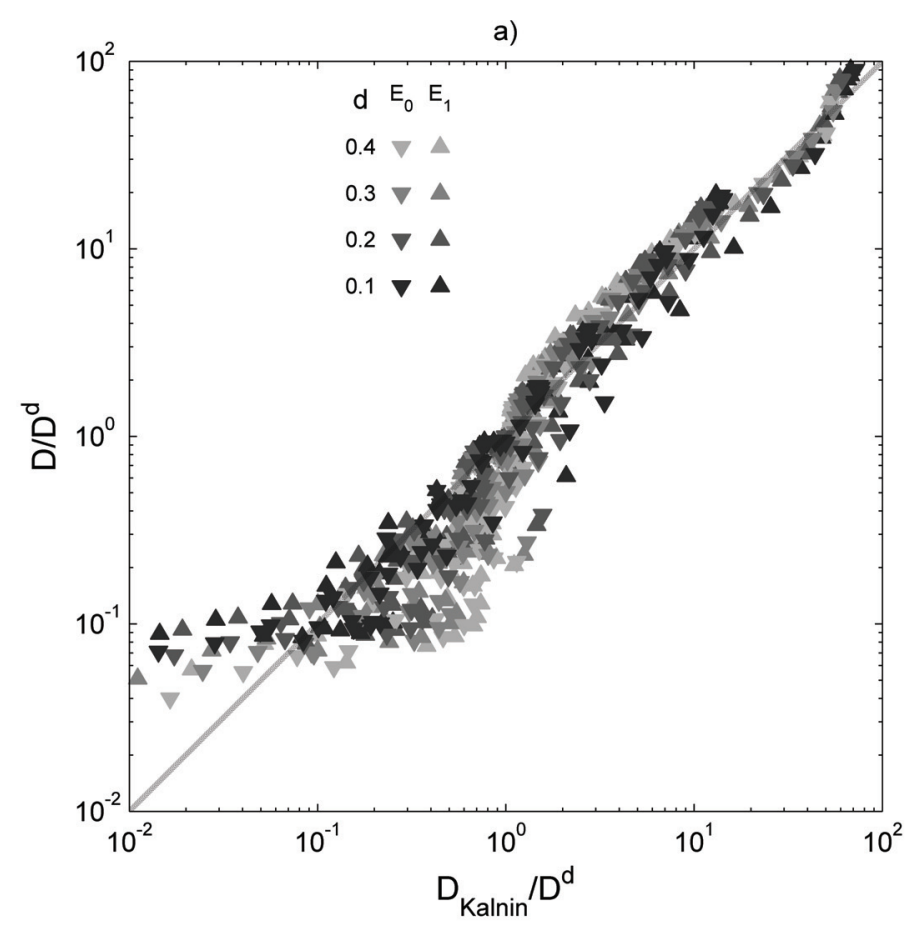

b)

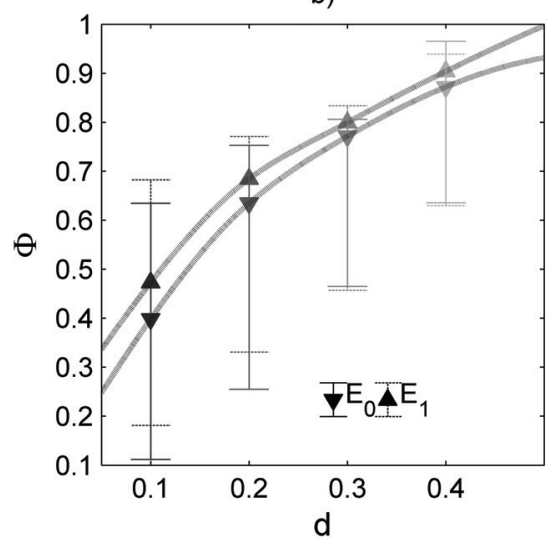

Fig. 6. a) Comparison between simulated results and the fitted algebraic model proposed par Kalnin and Kotomin (1998). The curve $\mathrm{y}=\mathrm{x}$ is plotted in dotted line. b) Values of the fitted parameter $\Phi$ in the Kalnin and Kotomin model by assuming it varies only with $d$. 95\% confidence intervals plotted as error bars were calculated by considering possible effects of $K$ and $r_{D}$. 
The two estimates of the effective diffusion coefficient were in good agreement. Significant negative deviations were nonetheless observed for the most heterogeneous morphology $\left(E_{1}\right)$. Besides, a higher bias was identified for small $D / D^{d}$ values related to either low $K$ or $r_{D}$ values (Fig. 6a).

To test whether observed discrepancies could be related to possible variations of $\Phi$ with $K$ or $r_{D}$, Fig. $6 \mathrm{~b}$ plots the previously identified values of $\Phi$ versus $d$ and their $80 \%$ confidence intervals (based on $10^{\text {th }}$ and $90^{\text {th }}$ percentile values) calculated by adjusting independently $\Phi$ from results simulated for similar $K$ and $r_{D}$ values. $\Phi$ was found to increase significantly and non-linearly with $d$. The contribution of $d$ was however as high as the other variations due to $K$ or $r_{D}$. As a result, $\Phi$ should be envisioned as a nonlinear function of $d, K, r_{D}$ and of the morphology. This possible extension of the model proposed by Kalnin and Kotomin (1998) was related to the possible contacts between globules and the confinement generated by globule aggregates.

\section{Conclusions}

This work illustrates how the simulation of random walks in the general framework of Langevin Dynamics can be used to calculate efficiently effective transport properties in complex systems as food products. The proposed methodology was applied to the calculation of effective diffusion coefficients in emulsions for a wide range of conditions: $K=\left[10^{-2}, \infty\left[\times r_{D}=\left[10^{-2}, 10^{2}\right] \times d=[0.1,0.4] \times\left[E_{0}, E_{1}\right]\right.\right.$. Only approximations exist for this general problem and only simulations make it possible to assess the subtle effects related to the morphology (density, connectivity) of the emulsion or to the transition between the Fickian regime and Knudsen regime in dense emulsions. The continuous implementation of motion equations and their interface conditions made it possible to handle in single formulation all phenomena. The reliability of the whole approach was examined in very detailed microstructure with variations between the smallest and the largest globules up to 4 magnitude orders.

The simulated results showed that $r_{D}$ had a major effect and controlled the magnitude order of $D / D^{d}$, while $K$ was not too small. For very low $K$ values, $K$ had a drastic effect and led to a confinement of trajectories on long terms. The effects of $r_{D}$ and $K$ did not commute since very low $r_{D}$ values restricted the trajectories within globules only during short or middle terms. For $r_{D}$ values lower than 1 , the possible connectivity between globules or the trapping of the continuous phase at high concentration of globules modified the diffusion paths so that the maximum $D / D^{d}$ value occurred for a $K$ value lower than 1. The core-shell model proposed by Kalnin and Kotomin (1998) was compared to our simulated results by adjusting the parameter $\Phi$ and could be proposed as a phenomenological estimate of effective diffusivities in emulsions. The background theory of this model should be however modified to account for variations of $\Phi$ not only with the globule densities but also with the complex morphologies and distributions of globules. Indeed, close to the percolation threshold, the local variations in the globules concentration modify the effective diffusion coefficient even for monodisperse emulsions.

Since our results rely on simplifications, which may be unrealistic for true macroscopic emulsions, further work is desirable to account for i) relative displacement or reordering of globules (e.g. due to Brownian motions) and ii) possible specific interactions at the globule interface due to the presence of surfactants. A 3D version of our code is yet available.

\section{References}

Belova, I. V., Murch, G. E., 2003. The effective diffusivity in two-phase material. Defect and diffusion forum, 218-220, 79-89.

Busch, K, Soukoulis, C. M., 1995. Transport properties of random media: a new effective medium theory. Physical Review Letters, 75(19), 3342-3445. 
Cranck, J. 1975. "Chapter 12: Diffusion in heteregeneous media." In The mathematics of diffusion, 2nd Edition, Oxford University Press, 266-285.

Cussler, E. L., 1997, Diffusion: Mass transfer in fluid systems, 2nd Edition. Cambridge University Press, Cambridge, 580p.

Doulia, D., Tzia, K., Gekas, V., 2000. A knowledge base for the apparent mass diffusion coefficient $\left(\mathrm{D}_{\text {eff }}\right)$ of foods. International Journal of Food Properties, 3(1), 1-14.

Kalnin J. R., Kotomon, E. A., Maier, J., 2002. Calculations of the effective diffusion coefficient for inhomogeneous media. Journal of Physics and Chemistry of Solids, 63, 449-456.

Kalnin, J. R., Kotomin, E., 1998. Modified Maxwell-Garnett equation for the effective transport coefficients in inhomogeneous media. Journal of Physics: Mathematical and General, 31, 7227-7234.

Küntz, M., van Mier, J.V.M., Lavallée, P., 2001. A lattice gas automation simulation of the non linear diffusion equation: a model for moisture flow in unsaturated porous media. Transport in porous media, 43, 289-307.

Marsh, C. A., Coveney, P. V., 1998. Detailed balance and H-theorems for dissipative particle dynamics. Journal of Physics A: Mathematical and General, 31, 6561-6568.

Nauman, E.B., He D.Q., 2001. Nonlinear diffusion and phase separation, Chemical Engineering Science, 56, 1999-2018.

Riley, M.R., Muzzio, F.J., Buettner, H.M., Reyes, S.C., 1994. Physical Review E., 49(4), 3500-3503.

Ronis, D., 1986. Diffusion in random two phase media. Physical Review A, 36(4), 1908-1928.

Snitzman, A.-S., 2001, On a class of transient random walks in random environment. The anals of Probability, 29(2), 724-765.

Zhu, J., Chen, L.-Q., Shen, J., Tikare, V., 2001. Computing the effective diffusivity using a spectral method. Materials Science and Engineering, A311, 135-141. 